disease to the extent of modifying, intensifying, or nullifying the morbid process, as soil influences the growth and results of the seed sown upon it. That the environment of the body may also potentially affect its vulnerability to or immunity from disease. That if the nature of the materies morbi of specific infective diseases be organic or microbic, such lowly organisms as have been causally associated with certain diseases are likely to be profoundly influenced by the soil upon which they are cultivated, and are therefore peculiarly liable to evolutionary changes in the course of their rapid propagation and multiplication. That the pathogenetic properties of such organisms or viruses may be acquired, lost, modified, or varied during their serial cultivation in living bodies or other culture media. That seeing that the possible modes of reaction of the living body to noxious agencies or influences are limited in number and nature, similar or identical symptoms may be occasioned by different pathogenetic causes; while the same pathogenetic agencies may occasion different symptoms in different persons. That the first line of defence against invasion of the human body by infective diseases is a sanitary environment, pure air and water, good food, clean clothes and houses, and that such environment not only permits but promotes healthy living, which itself tends to raise the normal resistant powers of the body and the blood.

When I promulgated such views in the eighties of last century I was told by my reviewers in the medical press that "such teaching runs counter to all the best work of modern times"; that I was " essaying with all the vigour of youth to demolish the theories of specific disease which have sufficed to satisfy the acutest physicians of this and every age" ; and I was pontifically warned that when I had reached the experience of those authorities whose opinion I condemned I should be less eager to adopt them. The sequence of events, however, has not borne out the vaticinations of my critical mentors. On the contrary, I could quote abundantly from the most recent publications vindication and confirmation of my views on specificity and evolution in disease of 40 years ago.

\section{Pathology of Contagia.}

It is common knowledge that bacteriology has failed to demonstrate microbic causes in some of the most typical infective specific diseases, and the view is now being accepted in some quarters that the intimate nature of contagia may be rather of the character of an organised or unorganised ferment, or something neither vegetal nor animal, on the borderland of the organic and inorganic, capable under suitable conditions of gathering pathogenic properties de novo and liable to specific differentiation, according to the soil upon which cultivation takes place. Light may not improbably be thrown on the intimate pathology of contagion by study not only of fermentive processes but of katalysis and contact actions, from which chemico-physics is deriving new and astonishing results.

The study of so-called "deficiency diseases," together with the vitamine hypothesis, has assisted in calling attention to novel and far broader views in æetiological pathology. The almost exclusive search for the last two or three decades of positive extrinsic causes, such as microbes, for all so-called specific diseases of man and animals has made it difficult for some minds even to contemplate a negative nutritional explanation of diseases with such characteristic symptoms as scurvy, beri-beri, rickets, pellagra, and kerato-malacia. It is therefore refreshing to find among orthodox pathologists the acceptance of such non-microbic causation of specific diseases. Even in disease in which the presence of a parasite is indubitable, the modus operandi may be both non-specific and yet highly characteristic. Take scabies, for example, with its polymorphism of papules, vesicles, excoriations, and pustules; do these phenomena constitute a specific disease? May not a similar question be asked in the case of some diseases which are also associated with vegetal or animal parasites?

\section{Conclusion.}

But to return to my theme of specificity and evolution in disease, of the man versus the microbe, or the importance of soil as well as seed; I rejoice to find in recent literature ample confirmation of my severely criticised thesis of 40 years ago. Writing on the trans. mutation of bacteria, Dr. S. Gurney-Dixon observes that "No single property of bacteria can be regarded as specific," and that the pathogenesis attributed to an organism may be dissociated from it. Again, almost repeating my words, he says : "Species of bacteria have developed from a common stock. In the case of some of them the differentiation dates from a remote past and the specific characters are comparatively fixed. In the case of others differentiation is of more recent date and the newly acquired characters are less permanent and 'reversion' in one or other character is more frequent." Yet again, " there is no reason to doubt and abundant evidence to support the opinion that in this field of life, as in others, the forces of natural selection and the survival of the fittest have been at work and have resulted, in the course of ages, in the evolution and differentiation of the various types of bacteria which we recognise and distinguish to-day. ...... It is reasonable to expect that amongst the bacteria natural variation would occur with greater frequency than amongst higher forms of life; ...... such variations would be more readily noted in their case, since as many as 30 or 40 successive generations may be observed in the course of 24 hours." Not only does the light of evolution help us to understand the genesis of specificity in disease, and to recognise the potency of environment upon health, of soil upon unicellular organisms associated by parasitism with pathogenesis, but also by realising that the metazoa, including mammals and man, are the "heirs of all the ages." This truth recalls to us the affinities of the highest with the lowliest whose cells have never entered upon colonial life, as seen in inflammation and reparation in neoplasms, benign and malign; in the case of the latter helping us to elucidate them by the study of embryonal reversion of cell-type and of bastard forms of conjugation and reproduction.

I close as I began by pleading for breadth of view and a wide and philosophic pursuit of the basic principles of our science and art. The wise pathologist should be a man "willing to listen to every suggestion, but determined to judge for himself. He should not be biassed by appearances, have no favourite hypothesis, be of na school, and in doctrine have no master."

\section{( $A N C E R$ OF THE COLON}

\section{ITS CAUSATION AND TREATMENT.}

BY SIR W. ARBUTHNOT LANE, BART., M.S. LOND., F.R.C.S. ENG.,

CONSULTING SURGEON, GUY'S HOSPITAL, ETC.

BEFORE passing on to consider the operative treat. ment of cancer of the large bowel, which is the subject of this discussion, it is of vital importance that we should, in the first instance, endeavour to obtain a thorough grasp of the factors which determine the development of cancer in this situation, not only with a view of arriving at the best methods of treating it when it exists, but especially for the much more important purpose of diminishing the incidence of this deadly scourge, which apparently claims a larger number of victims every year. If we can obtain a clear idea of the conditions which antedate and produce cancer of the large bowel we may be in a position to control or prevent its development. Is any problem more important to the community or more interesting to us as individuals than this?

Crusal Factors of Cancer in the Large Bowel.

The causal factor which is common to cancer in any part of the body is "traumatism," using the word in its widest sense. Another factor is a degenerative change in the tissues of the body, brought about in the vast majority of cases by the products of intestinal

1 Comprising remarks on the discussion of the Surgical Treatment of Malignant Disease of 
auto-intoxication or by syphilis, or by both together. The third factor $I$ will call $x$, since it is at present unknown to us. It may be an organism, but we are entirely in the dark as to its nature and behaviour. Although we are ignorant of the nature of this agent, we are justified in assuming that it does not come into action except in the presence of the results of the traumatic and degenerative factors without which it cannot obtain a foothold in the tissues.

Can we prevent the incidence of $x$ by removing the traumatic and toxic factors on which it depends" This would seem to be distinctly probable.

How can we avoid the effect of the traumatic and toxic factors of chroric intestinal stasis and syphilis? I believe that if the State were sufficiently alive to the interests of the community to take the necessary action syphilis might be very materially reduced and possibly finally eliminated altogether by legislation. It is also probable that much of the degenerative change that ensues from syphilis might be modified or even avoided by early and efficient treatment. I will not refer more fully to the influence exerted by syphilis in the production of cancer, but will endeavour to show how the traumatic and toxic effects of intestinal stasis manifest themselves, and in that way to demonstrate the means necessary to eliminate their influences.

Let us take these factors in their order. How far can we influence the most important of these-namely, traumatism?. The gastro-intestinal tract affords an excellent illustration of the manner in which traumatism produces ulceration and cancer. It is necessary for the development of cancer that the ulcer should be sufficiently chronic. But as regards the tongue, the pharynx, the csophagus, the lesser curvature of the stomach and the immediate vicinity of the pyloruscancer develops in just such spots as are constantly exposed to injury, either in the form of impact or strain. The same applies to the incidence of cancer in the large bowel, but this we will consider more fully later. It is clear that if we can obviate the irritation of the tongue by a ragged tooth or other source of traumatism we can certainly prevent the development of cancer in this situation. We know also that if such an irritant is left to exert its baneful influence for a sufficient length of time it will almost certainly produce cancer. The development of cancer in the tongue and elsewhere is therefore largely a question of time. The same applies to cancer of the cesophagus, stomach, and large bowel, in which, however, the mechanical conditions are somewhat more complicated than in the case of the tongue.

As regards the intestinal tract, the mechanical factor is bound up intimately with the toxic factor in the condition to which I have given the name of chronic intestinal stasis. Can we learn anything from the immunity of the duodenum and small intestines from cancer? In this portion of the alimentary canal, and especially in the duodenum, we have in action the several factors that determine the presence of cancer in the rest of the intestinal tract. They are the mechanical factor, the toxic factor, and presumably the factor which I call $x$. That the last factor is anable to exert its influence upon an ulcer which is often very chronic in this locality cannot be due to the presence of bile or of the pancreatic secretion, since cancer develops freely in the ducts of the organs secreting these juices. Infective organisms may flourish in the diseased duodenum. Why is that which produces cancer in abeyance? Dr. N. Mutch reminds me that the area of freedom from the effects of the factc $x$ corresponds to the distribution of enterokinase, the ferment of ferments which activates the precursor of trypsin, the secretion of the pancreas. It exists in quantity in the duodenum and in a diminishing amount through the small intestine. Has this material any influence on the factor $x$ ? If not, what is the agent that prevents it exerting its usual effect in this situation? A study of the reason for the freedom from cancer of the duodenum, and to a lesser degree of the small intestine, may lnufold the secret of the mode of action of $x$, the unknown factor, and may throw some light on the treatment of this disease.
In chronic intestinal stasis there are two distinct factors, the primary, which is simply and solely mechanical; and the secondary, which is essentially toxic and results from the effects of the mechanical or traumatic factor.

I am assuming that the reader is familial with the condition I call chronic intestinal stasis, since I have described it so fully on many occasions during the last 20 years. The behaviour of the individual to stasis varies enormously. This variation depends chiefly on the resisting power of the subject at a time when first exposed to its influence. The vitality may be reduced by bad or unsuitable food, unsatisfactory hygienic surroundings, exposure to cold, \&c. In the face of the very different opinions that are held on the subject of the treatment of stasis it is most important that we should be thoroughly familiar with every detail of the varied aspects it presents.

Stasis in a Patient with Little Vitality.

Perhaps I can put the case best by describing extreme degrees of this condition.

Take the patient with little vitality who makes no attempt to form resistances or retaining membranes, whose pelvic colmn elongates enormously, whose iliac, descending, and transverse segments of the large bowel become progressively elongated, tortuous, and prolapsed, and whose cæcum and ascending colon dilate and prolapse considerably. The absence of any acquired membrane or crystallisation of lines of force tending to obviate or control the prolapse of

FIG. 1.

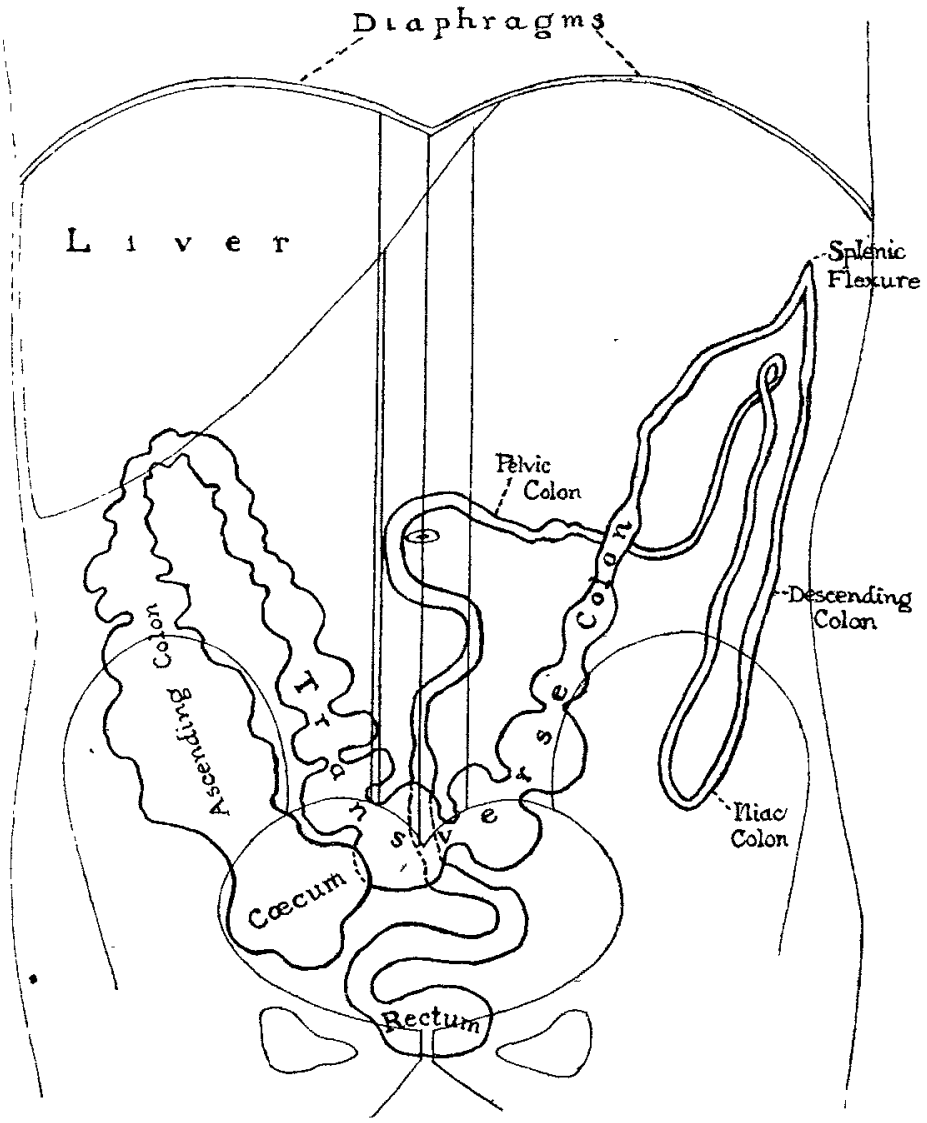

Diagram, by Dr. Jordan, showing the radiographic appearances of an extreme case of chronic intestimal stasis in the first group. I was taken while the patient was in the recumbent posture. It is
sufficiently clear to require no further detailed description.

the several portions of the bowel is the marked feature of this variety. There is no ileal kink and probably no control by the appendix. The small intestines are dilated and their walls are thin and Haccid. If the duodeno-jejunal angle is at all abrupt the duodenum is very widely dilated in its entirety, and its walls are soft and inelastic. The pylorus is but moderately spastic and the stomach is dilated, perhaps to a considerable extent, with little or no evidence of hyper. trophy of its walls. This explains the want of success of gaistro-enterostomy alone in the treatment of this condition There is very rarely any suspicion of duodenal or of gastric alceration. The acquired band which anchors the gall bladder to the prlorus, duodenum, and transverse colon is not developed, and gall-stones rarely complicate the condition.

In this type auto-intoxication is the marked feature abdominal symptoms, except for constipation and 
flatulence, being absent or calling for comparatively little serious attention, unless perhaps in the cæcum, ascending colon, and part of the transverse colon, the mucous membrane of the large intestine is inflamed to a varying extent, while the associated spasm of the muscular wall reduces its lumen, and allows of the passage of the intestinal contents through the bowel and over the inflamed mucous membrane in limited quantities only to be evacuated with great difficulty through the obstruction formed by a prolapsed elongated pelvic colon. This is shown clearly in an ordinary typical case in Fig. 1.

This is the condition for which surgeons have, as they suppose, modified and improved on the operation of colectomy, which I suggested for it, by effecting a removal of the croum and ascending colon, calling it a hemi-colectomy. They excise that portion of the large bowel in which are accumulated the fæcal contents whose passage through the rest of the colon is obstructed by the elongation, angulation, and prolapse of the pelvic colon and by the spasmodic contraction of the muscular wall of the descending and iliac colon, due to the infection and inflammation of the mucous membrane lining it. Can any procedure be more illogical?

Assuming my description of the mechanics of this condition to be accurate, and radiography has rendered it perfectly capable of ocular demonstration, what is one to think of the attempts to meet the situation by operations performed with the object of suspending or fixing the dilated, over-distended cæcum, or of shortening the mesentery of prolapsed partions of bowel, or of the fixation of the mesentery to the anterior abdominal wall, or of excision of part of the elongated transverse colon? The only excuse for the adoption of such methods is a want of familiarity with the physiology of chronic intestinal stasis and with its mechanics. The reasoning person will ask himself, Why are the cæcum and ascending colon distended, dilated, and prolapsed? The answer is perfectly obvious: they are in this condition because of the obstacle to the passage of their contents which exists in the bowel beyond. He will then naturally wonder by what means the removal of the sac containing the dammed-up contents of the cæcum and ascending colon can be benefited by their excision. He would argue that the obstruction in the remaining portion of the bowel could in no conceivable manner be affected by his surgical procedure. He would realise that the removal of this sac would only entail the further damming back and infection of the contents of the small intestine, with disastrous results to the individual.

The same criticism applies equally to the less ,radical procedures of suspension as initiated by Rovsing of Copenhagen in 1906. His treatment was adopted and modified by Coffey and other distinguished surgeons. If additional proof were required of the truth of this it is afforded by the fact that if after a colectomy the patient is allowed to become constipated habitually for a long time, the residue of pelvic colon left at the operation elongates and may readily reach the great length it possessed before the operation, with all the associated disabilities. It again serves as an obstacle to the passage of material from the ileum, material is dammed back and the mechanical and toxic conditions which existed previously reappear. This can only be met by the excision of as much as possible of the elongated pelvic colon, and by ensuring that in the future this portion of the bowel shall not be overloaded and overdistended beyond its capacity. It is obvious that the small residual portion of colon left after a colectomy is not of a calibre sufficient to accommodate the products of 24 hours' digestion, and to ensure perfect drainage it must be evacuated two or three times a day.

The only rational means of meeting the obstruction in the large bowel and of freeing the ileal effluent is by the removal of the large intestine to the level of the pelvic colon. Not only is the entire inflamed and elongated portion of the bowel removed together with the part which has become over-distended, but the extremity of the ileum is inserted directly into the lower limit of an elongated pelvic colon. In certain circumstances it may not be possible to remove the bowel, but the ileal effluent may be freed as effectually by dividing the ileum and putting it directly into the pelvic colon. The objection to such a procedure is the possible regurgitation of material into the large bowel, and for this reason colectomy should be performed when feasible. The regurgitation may be controlled by the evacuation of the contents of the pelvic colon two or three times a day.

Stasis in a Patient with Great Vitality.

In the other extreme the symptoms of chronic intestinal stasis which result are mainly mechanical, autointoxication being a less conspicuous feature of the condition.

The patient is strong, vigorous, and full of fight. The delay of material in the large bowel is met by the abundant formation of bands and membranes in certain situations which are definitely localised, where they represent the

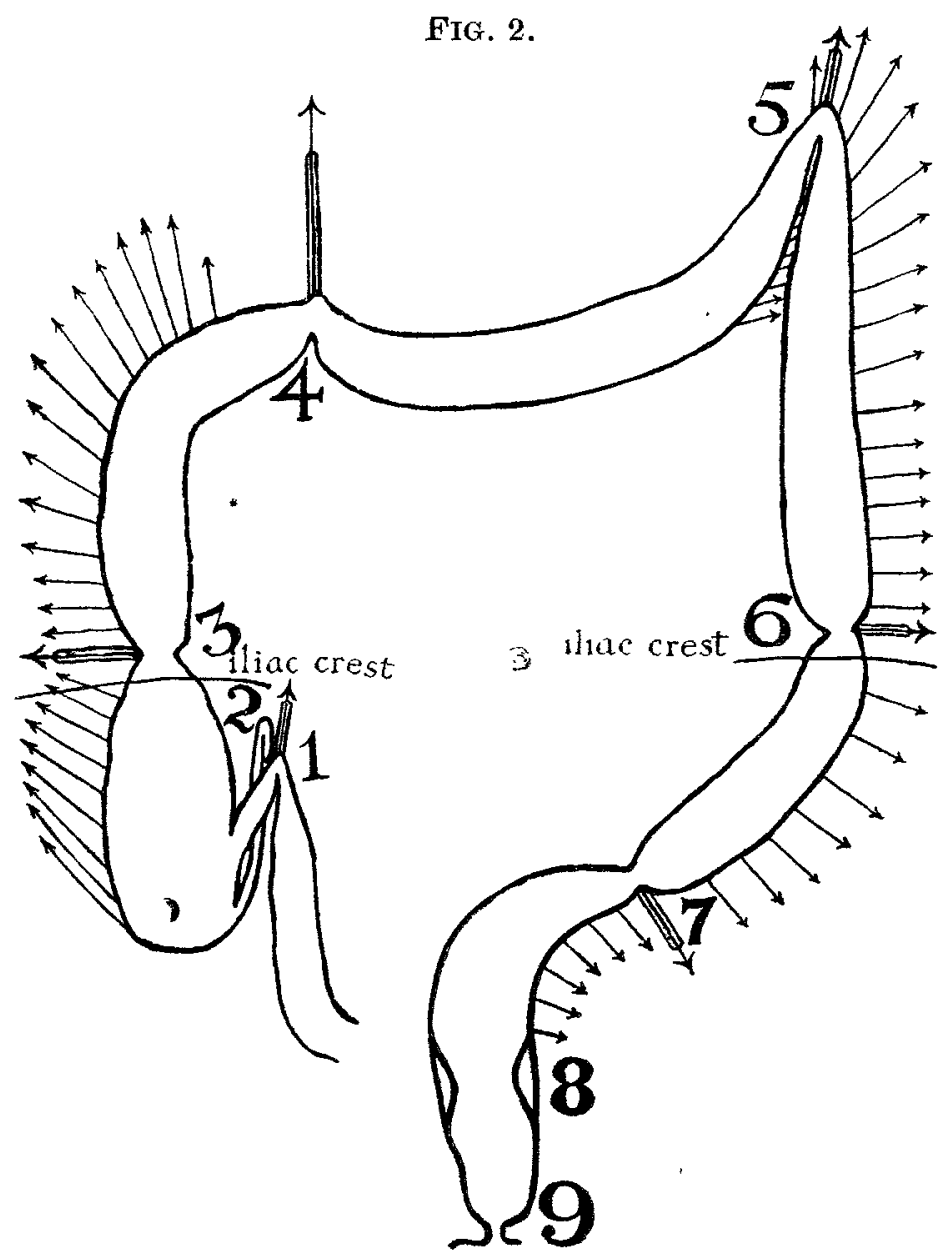

The arrows show the direction of the lines of resistance. 1. Indicates the ileal kink. 2. The appendix passing up behind the ileum and controlling its lumen in the erect posture (it is secured by eyolntionary bands to the back of the mesentery and is itself kinked and its distal portion obstructed by the anchoring adhesions). 3. The strong band which obstrncts the lumen of this portion of the colon. 4. The band which extends down from the liver, gallbladder, pylorus, and duodenum. 5. The splenic flexure whose angularity is accentuated and whose lumen is correspondingly diminished. 6. The deformity of the descending colon by a band similar to 3. 7. The last kink. 8. The circular band of muscle fibre described by W. J. Mayo. 9. The internal sphincter.

crystallisation of lines of force. I have explained the mode of formation of these bands so clearly on many occasions that if their causation has not been grasped $I$ feel that any repetition on my part will serve no useful purpose.

The first to appear is the thickening on the under surface of the mesentery at the junction of the iliac and pelvic segments of the colon. This membrane develops and at a later period diminishes the lumen of the bowel at this point progressively, and consequently obstructs the passage of the facal matter through it. This particular band I call the "first and last kink," the first as it is the earliest to appear and the last because it is the lowest of the several kinks in the drainage scheme. Secondary to the obstruction exerted by this kink bands develop in the peritoneum on the outer aspect of the descending colon. The direction of the controlling bands varies at different points of the bowel. Those supporting the splenic flexure are nearly vertical. They angulate it and 
reduce its calibre. Those attaching the descending colon immediately above the iliac crest are transverse, and often so strong as to deform and constrict this portion of the bcwel in a very marked manner. Except for the acquired band which extends from the gall-bladder to the pylorus, duodenum, and transverse colon a little to the left of the hepatic flexure, the transverse colon receives no definite additional support from any acquired membrane. This band which extends from the gall-bladder tends to diminish the lumen of the large bowel at its point of attachment to it, and later to produce a considerable amount of obstruction. Similar acquired membranes develop on the outer side of the peritoneal reflexion from the cæcum and ascending colon. Those about the hepatic flexure are almost vertical. They exert but little control on the flexure, and do not materially affect its angulation. Their direction gradually changes so that just above the iliac crest they are arranged transversely, and in this situation may form a very strong band which van materially drag on and obstruct the lumen of this portion of the bowel.

Below the level of the crest the acquired bands rapidly become more vertical, forming a membrane of varying strength surrounding and securing the cæcum. Like all the other acquired membranes, its function is to afford a resistance to the downward displacement of the viseus to which it is attached. A controlling appendix or a well-marked ileal kink is frequently present. If the duodeno-jejunal junction is acute the duodenum, and especially its first part, is moderately dilated, its muscular wall hypertrophied, and its mucous membrane perhaps ulcerated. The pylorus is hard and contracted, while the stomach is usually somewhat dilated and its muscular coat hypertrophied. An ulcer may be present at the pylorus or in the lesser curvature, the situation varying with the stage of the disease. In this type the mucous membrane of the large bowel is not inflamed, so that material tends to accumulate behind the several acquired points of obstruction in an amount varying with the extent of the diminution of the lumen of the intestine.

Between these extremes of chronic intestinal stasis innumerable intermediate stages and combinations exist, the conditions present varying with the degree of vigour or fighting power of the individual and with the state of auto-intoxication.

\section{Diverticulitis.}

The type may be modified by the abundant deposit of fat in the tissues. As the result of this distension

FIG. 3.

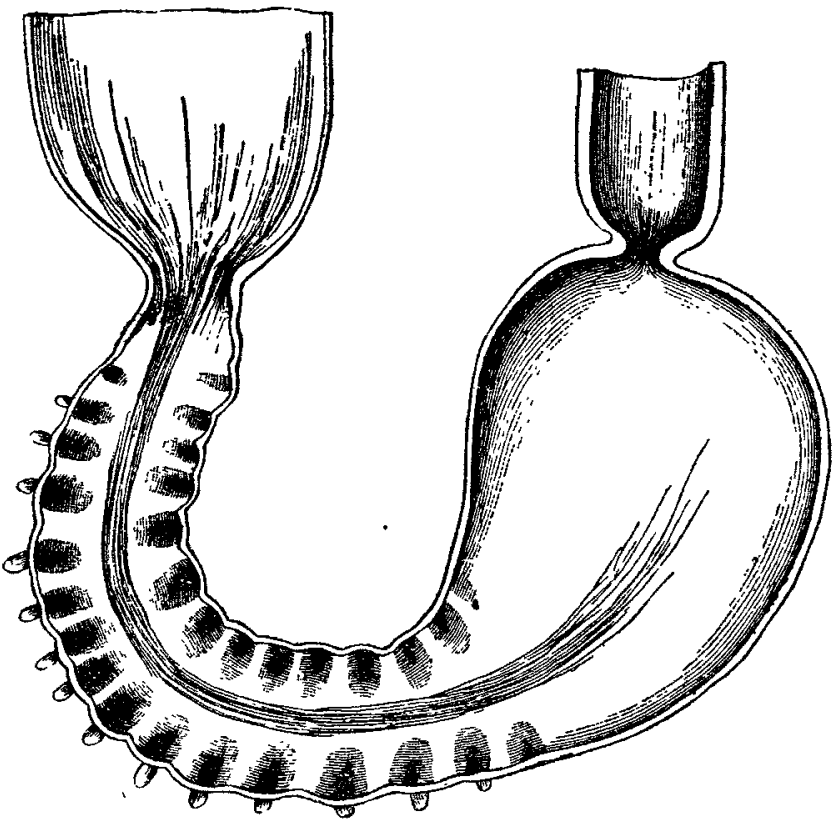

This diagram, which appeared in illustration of a letter to THE LANCET by the author (1920, i., 221), represents a loop of large intestine laid open. It lay in a hernial sac. Its mesentery diaty proximal to the point where it is obtucted is uniforming diated proximal to the point where it is obstructed is uniformly che They are trophied band of longitudinal muscle fibres.

changes may be observed in the large bowel proximal to any point of obstruction, not in the immediate vicinity of the obstruction where the bowel is uniformly dilated, but at an interval. The changes consist in the forma. tion of hernial protrusions of mucous membrane through the muscalar coat of the bowel. Later freal concretions may form in these diverticula. Infective processes may result and subsequently cancerous foci may form in the bowel damaged by traumatism. This condition is called diverticulitis, and it is identical in its causation with the sacculi which form in the bladder wall. I described it and its causation in a paper in the Guy's Hospital Reports, 1885, under the title of "Pathology of Extravasation of Urine." A brief abstract of the portion relating to diverticulitis was published in THE LANCET, Vol. I., 1920 , p. 220 , with an illustration showing the mechanical condition. (See Fig. 3.)

\section{Radiological Evidence.}

Here I would like to call attention to the excellence of the radiological work extending over a period of many years, which Dr. A. C. Jordan has äone in the patient study of the condition of the intestine in chronic intestinal stasis. He has thrown a flood of light upon the mechanism of the gastro-intestinal tract and has made many original observations on the subject. He has demonstrated, by means of the bismuth meal with radiography, all the changes which I have described in the clearest manner possible, so that they are obvious to the most superficial observer. I attribute his remarkable success to the fact that he made a point of being present at the operations on a very large number of cases. In this way he was able to elaborate his methods and to clear up many difficult points in the radiology of stasis which could not have been effected in any other manner. I am greatly in his debt for his invaluable help in confirming my views on the causation of the changes in stasis.

Different Frequency of Cancer in the Two Extreme Types.

A matter of great importance is that those cases included in the first group of stasis do not get cancer of the large bowel any more than they get carcinoma of the stomach and œsophagus, while those in the second group axe very liable to the formation of cancer at any of the points of obstruction produced by the acquired bands or in the situation of the muscle at the junction of the pelvic colon and rectum, to which Dr. W. J. Mayo called attention, and about the sphincter ani. Another point is that the consistence of the contents of the large intestine varies greatly throughout its length, while in the cæcum and ascending colon it is of a fluid or pasty consistence, in the descending iliac and pelvic portions of the colon it is usually firm and may be very hard. Therefore the physical character of the freal matter is a matter of importance in the consideration of its effects upon and its capacity to pass through any portion of the bowel whose lumen is constricted to a material extent. The explanation of the different frequency of cancer in the two extreme types is obvious. To exert traumatism at the seat of constriction it is necessary that there should be sufficient thrust produced by the muscular wall of the bowel, and again the mechanical effect of that thrust varies directly with the bulk and consistence of the portion of the intestinal contents which is forced against the partly obstructed segment of bowel, and with the degree of the obstruction.

In the first group the very elongated bowel with its feeble muscular wall, with its inflamed mucous membrane and its very scanty contents exerts but a slight effect upon the mucous membrane at the junction of elongated loops of colon, the kinking or obstruction by membranes being non-existent in these cases. Instead of developing excessive power by the hypertrophy of the muscle coat of the bowel the proximal colon gives up the struggle readily, dilates and prolapses. In the second group the bowel is not elongated, its wall is hypertrophied, the freal contents are abundant and bulky, and the obstruction is acute and well defined. In the first group the enfeeble ment of the muscle coat of the bowel resulting from the extreme degree of intestinal auto-intoxication eliminates the mechanical factor which in the case of the large bowel is responsible for the formation of cancer, but by causing degenerative changes in the breasts, uterus, ovaries, and other organs, it renders them much more liable to cancer than the second group.

It is of interest to note that this association of cancer with the cystic degeneration which results in the breasts 
from chronic intestinal stasis is now being gradually accepted by the profession. It is many years since I called attention to it in the clearest manner possible. The same applies equally to the ovary, pancreas, liver, and other organs. Therefore it is obvious that the mechanical factor in "chronic" intestinal stasis plays a vastly more important part in determining the presence of cancer in the large bowel, as it does in the stomach, than does the toxic factor.

Possibility of Eliminatıon of Mechanical Causal Factors.

Again the question arises, Can we eliminate the mechanical factors which determine the development of cancer in the large bowel? These factors are : (a) the constriction of the lumen of the bowel at several points by the formation of acquired bands or by the spasmodic contraction of a hypertrophied sphincter muscle; (b) the thrust of the hypertrophied muscle coat of the bowel proximal to the obstruction; $(c)$ the increasing desiccation of the contents of the large bowel.

Dr. Hoffman has shown in his elaborate work ${ }^{2}$ on the causes of death that cancer is increasing with great rapidity in civilised communities. He points out that while it apparently does not exist in savage life, it occurs just as frequently in the civilised savage as it does in the white man. The same would appear to apply to many of the lower animals in the wild state and in captivity.

Dependence of cancer upon stasis.-Stasis can be avoided by attention to the diet of the individual from birth, paraffin being used before meals so as to meet the unavoidable disadvantages of the mechanical relationship of the individual to his surroundings that civilisation entails. By mechanical relationship to surroundings I mean the very different and varied attitudes and exercises that are employed by the savage as compared with those of the civilised individual. For example, in civilisation the trunk is erect from morning to night, the recumbent posture being only assumed at night time. How different are the conditions in savage life !

That paraffin is of incalculable use in facilitating the passage of the contents of the intestine through a hampered pylorus, duodeno-jejunal junction, and terminal ileum is well recognised by the profession. By rendering the contents of the colon more fluid and by lubricating the mucous lining it obviates the formation of bands and spasmodic contraction of involuntary muscle fibres, diminishes the necessity for the thrust on its contents exerted by the muscle wall of the bowel, and influences very materially the character of the impact of the fæces which are no longer hard and desiccated upon any point of constriction in the lumen of the large bowel. In other words, if you wish to avoid cancer of the large bowel use enough paraffin to render the fæcal matter as it is expelled from the anus soft and non-irritating. Paraffin has afforded more comfort to the community and has prolonged life and avoided the development of disease more effectually than any other material of which I am aware. Further evidence of the dependence of cancer upon intestinal stasis is shown by what might be regarded as an intermediate state of civilisation from the physical standpoint, as in such countries as India and China.

Locality of the growth.-Cancer develops in the large bowel at one of the points of constriction by acquired membranes or at the rectal sphincters. The exception is in the case of diverticulitis, in which the malignant growth arises in an area of the bowel proximal to the seat of obstruction and not at the point where the constriction exists. The treatment of carcinoma of the rectum has been excluded from this discussion, so I will not refer to it beyond stating that while in my experience no single operative procedure best meets the conditions present in all cases of cancer of the rectum and pelvic colon, the operation which I find the most effectual in picked cases involves the complete removal of the disease by the excision of the rectum and pelvic colon and all infected soft parts and glands. 2 The Mortality from Cancer throughout the World, by F. I.
It was described in the Clinical Joumal, 1907 and $190 \mathrm{n}$, in a paper entitled "The Operative Treatment of" Cancer of the Large Bowel," and in THE LANCET, 190\%, vol. i., p. 418, Clinically cancer of the large bowel is not usually recognised till the symptoms which indicate a varying degree of obstruction call the attention of the surgeon to the seat of progressive diminution of the lumen produced by the growth.

As regards the locality of the tumour the common situations are in the points of the bowel where its lumen is constricted by acquired adhesions or by muscular spasm-viz. : (1) In the ascending colon at or about the level of the crest of the ilium; (2) in the transverse colon to the left of the hopatic flexure where the lumen of the bowel is constricted by the acquired band which extends down from the gall-bladder, liver, and pylorus; (3) at the splenic fiexure; (4) in the descending colon at or about the level of the crest; (5) in the colon at the junction of the pelvic and iliac segments in the situation of the last kink; (6) about the rectal sphincters.

\section{Treatment}

The treatment of cancer of the colon must differ with the locality and extent of the growth, the condition of the intestines at the time of the operation (especially as regards accumulated contents), the vitality of the individual, the invasion of adjacent viscera or abdominal wall, the presence of secondary growths, the degree of intra-abdominal tension, the age and sex of the patient, the amount of fat present in the tissues, and the circumstances in which the operation is performed.

There is one point of great practical interest in the operative treatment of cancer of the big bowel, and that is the rarity with which peritoneal adhesions form subsequent to operation. This would seem to be due to the fact that owing to the gradual diminution of the lumen of the bowel by the growth, the muscle coat of the intestine proximal to it becomes greatly hypertrophied, so that the bowel is capable of vigorous action and in a certain proportion of cases a variable amount of fluid lies free in the peritoneal cavity. mention this as it eliminates to a very great extent the risk of adhesions which exists after all abdominal operations of any extent in which there has been no local obstruction of the lumen of the bowel and corresponding hypertrophy of its wall. This risk of the formation of inflammatory adhesions is exaggeratec in the flaccid, inert, wasted intestine present in the simple or toxic variety of stasis.

Advantages of colectomy.-A careful study of the causation and the mechanics of stasis on which cancer depends would indicate clearly that the ideal operation for cancer of the large bowel is colectomy, providing that a sufficient length of pelvic colon can be secured into which the end of the ileum can be inserted directly. and that other conditions are favourable to its performance. The advantages which this procedure afford are as follows:-

(1) A perfectly secure junction of the end of the ileum to the pelvic colon can be readily effected; if this operation be performed skilfully the risk of leakage of the intestinal contents is infinitesimally small, providing that the patient has any residual power of repair.

(2) The ileal contents are evacuated at once and freely by means of a large oesophageal tube (usually No. 25) passed up through the anus for about six inches above the junction. The tube is left in position for six days, by which time firm union has taken place at the junction. I have employed this method for more than 20 years, and I can onl yrecall one casenamely, that of a young child-in which the end of a tabe produced any injury to the bowel, and that patient was in an apparently hopeless condition at the time of the operation. The contents of the ileum being fluid, and their consistence being rendered still more so by the free admixture of paraffin administered both by mouth and through the rectal tube, no such clogging occurs as too frequently takes place when a large tube is used in a similar manner for resection of a growth in the continuity of the large bowel.

(3) If, as is too frequently the case, a large quantity of material has collected in the colon behind the obstruction it is removed in its entirety and the system is at once freed from the deleterious effect of the poisoning it produces, as well as from its mechanical results.

(4) The abdomen is left quite flaccid after the operation, all intra-abdominal tension being relieved by the removal of the 
length of gut and its accumulated contents. It is difficult to exaggerate the importance of this factor of diminished intra-abdominal tension not only as regards the immediate reduction of the risk to life, but also as affecting the comfort and ease of convalescence.

(5) The prompt relief of the ileal stasis and of the consequences, which are the result of the damming back and infection of the contents of the small intestine, plays a very obvious part in the restoration of the patient to a state of health and vigour not enjoyed for many years.

(6) In the case of cancer in any situation other than about the last kink the resection of the colon removes the risk of orowh taking place at a later date in this last position. This risk is not a small one. I have removed the crecum, ascending and transverse colon for extensive carcinoma of the ascending colon, and have seen the patient ten years later with a primary inoperable growth in the situation of the last kink. In this case the ileum was put into the end of the transverse colon so that the last kink still remained operative as the obstruction responsible in the first instance for the growth in the ascending colon.

(7) Colectomy enables the operator to deal effectually with any secondary glandular infection far more thoroughly than is possible in a partial resection, since all anxiety as to the interference with the circulation of the residual bowel is removed by its excision.

18) In a fat patient if the growth is in the ascending or transverse colon the risk may be somewhat reduced by dividing the transverse colon to the left of the middle line, excising the proximal large bowel, and putting the end of the ileum direct into the side of the pelvic colon. Against this is the time taken in closing the distal end of the colon and the possibility of regurgitation.

Unless the surgeon is perfectly familiar with the mechanics of the large bowel in chronic stasis he may experience on the first occasion he performs colectomy sufficient difficulty in the removal of the colon as to render it a very serious operation. A knowledge of the arrangement of the acquired bands affords the operator a ready means of removing the bowel quickly, easily, and with reasonable safety.

The varying conditions which cancer of the large bowel presents make its treatment of the greatest interest to the surgeon. The disgust and annoyance caused by a colostomy, together with the trouble it entails on the patient and others, render it advisable that the surgeon should not hesitate to take much risk in avoiding its performance. The dread that the public has of infection is another strong argument against rendering the existence of cancer conspicuous by a colostomy. The operative procedure necessary to avoid a colostomy may sometimes make great demands on the skill, ingenuity, and judgment of the surgeon. Very much depends in most cases on the condition of the pelvic colon and on the possibility of inserting the divided end of the small bowel into it well beyond the growth.

Extensine Scope of Colectomy.

Perhaps nothing is so surprising as the remarkable and prolonged benefit that will frequently follow an operation on an irremovable carcinoma of the bowel by the complete diversion of the fæcal stream from it. One has also to realise in performing short-circuit operations that life can be carried on in apparently perfect health with only a short length of small intestine. The surprises which extensive operative measures may afford are often extraordinary, and unless there is evidence of secondary growth in important organs there is practically no limit to the field of operation. No procedure should be considered too radical or too extensive in cases in which adjacent viscera have become involved in the growth, and the more familiar the surgeon is with intestinal surgery the more readily will he carry out operations on conditions which may at first sight appear to be hopeless and inoperable.

Only on one single occasion have I excised the large bowel in its entirety. The operation was done on a patient who appeared to have a malignant growth obstructing the iliac colon. Eleven years previously her left breast had been removed for cancer. On opening the abdomen a mass was seen obstructing the iliac colon. It was peculiar in that it did not surround the bowel, but invaded it along its mesenteric attachment. While freeing the diseased bowel I found that a similar growth extended in varying amounts along the entire mesentery of the large bowel, even into the pelvic colon. I therefore proceeded to remove the entire colon, including the anal aperture. The end of the ileum was then sewn to the margin of skin which originally surrounded the anal orifice. The late $\mathrm{Mr}^{\mathrm{r}}$. Plimmer, with whom I discussed the case, was quite familiar with the pathological condition of the bowel, which he regarded as being secondary to the breast infection. The patient lived for some time and died from secondary growths, which were not observed at the operation.

In a certain number of cases of acute obstruction in which the patient's condition is such that any extensive procedure is inadmissible, the formation of a lateral anastomosis between the large bowel proximal to the growth and that distal to it, may be effected if the proximal bowel is not too distended and its wall too fragile. This does not free the growth from a certain amount of irititation from the fæcal contents, since the contents of the bowel always tend to follow the normal course. Later is may be possible to remove the large bowel without the risk of infection and the added difficulty which a temporary fistula would afford. Again, in the same condition it may be advisable to divide the ileum and to put it directly into the pelvic colon, closing its distal end or draining it should the colon proximal to the growth be distended with fæces. Later the colon can be removed if possible.

If the growth is in the splenic flexure, unless the conclition of the parts is such as to render a resection easy and safe, colectomy is the safest procedure. If the cancer is in the vicinity of the last kink and the condition of the growth and bowel is very favourable for resection this may be the safest course. But if the growth is of such a nature that to establish continuity of the divided ends of the colon would involve the patient in great risk, and especially if much material is dammed up in the colon, colectomy offers by far the best prospect of success. It is so much easier and safer in the large majority of cases to put the end of the ileum into the divided end of the pelvic colon in a colectomy than it is to join together the extremities of the large bowel after the excision of a growth. The risk of recurrence is also greatly diminished by the more extensive operation.

In women, owing to the greater area of the abdominal cavity, colectomy is performed with much more facility than in the male. The degree of intra-abdominal tension and of laxity of the abdominal wall are very important considerations in deciding on the nature of the operation. With a loose abdominal wall in a woman advanced in years colectomy affords a minimum of risk. When there is a very considerable amount of intra-abdominal tension at the end of the operation it is advisable to make no attempt to bring the edges of the muscle wall together but merely to suture the margin of the skin incision together by means of such a suitable arrangement of sutures as will prevent the escape of the intestinal contents. In this manner the area of the abdominal wall along the length of the incision may be increased for a breadth of about four inches, and it may be rendered still mole extensive and the intra-abdominal tension correspondingly diminished by undercptting the skin. By reducing tension in this manner a large number of lives have been saved which would have been lost had the muscle edges been brought together. As was pointed out earlier, intra-abdominal tension is a very potent factor in produsing obstruction, especially at the duodeno-jejunal junction.

Ex-SERVICE TUBERCULOUS PATIENT IN AIIERICA.- Surgeon-General H. S. Cumming, of the U.S Public Health Service, has arranged for a number of specialists in tuberculosis, not members of the Public Health Service, to visit the service hospitals, which contain 15,000 ex-Service tuberculous patients, and to study the conditions with a view to standardisation and improvement. Dr. David Lyman, of Wallingfor, Conn., Dr. Victor: Cullen, of Maryland State Sanitarium, and Dr. Martin E. Sloan, of Towson, Md., will officiate in the Eastern States; Dr. George $T$. Palmer, of Springtield, Ohio, in the Central States; and Dr. Henry Hoagland in the South-Western States. About two weeks will be spent in each hospital. 